\title{
ELECTROCARDIOGRAMA FETAL (E. C. G. F.) EN LA CONSULTA PRENATAL *
}

\author{
Doctores: Alfonso Jubiz H. $* *$ \\ Ignacio Escobar M. *** \\ Iván Jiménez R. $* * ; *$
}

\section{Introducción}

El electrocardiograma fetal (E. C. G. F.) fue encontrado por primera vez en 1906 por Cremer, utilizando un galvanómetro de cuerda, mientras efectuaba el registro de un electrocardiograma normal en una paciente con embarazo a término. Desde entonces el método se ha perfeccionado con los nuevos sistemas de registro.

Actualmente se utiliza en varios centros obstétricos del mundo para valorar el estado del feto in útero. Nosotros hemos puesto en práctica este método por primera vez como auxiliar en la consulta prenatal, con el objeto de valorar el estado fetal, hacer diagnósticos diferenciales con otras entidades obstétricas como la Mola y diagnóstico del embarazo en sus primeros meses.
Este es el informe preliminar de los resultados obtenidos en el presente estudio.

\section{Material y métodos}

Este trabajo se inició en el mes de agosto del presente año, en el Departamento de Fisiología, de la Facultad de Medicina de la Universidad de Antioquia, estudiando hasta el presente 25 pacientes pertenecientes al Servicio de Obstetricia del Hospital Universitario San Vicente de Paúl.

Los registros se han efectuado utilizando un electroencefalógrafo GRASS mode!o 111 , de 8 canales. Los electrodos empleados por nosotros fueron del tipo común para electroencefalografía, los cuales se pegaron a la pared abdominal por medio de una pasta conductora, previa limpieza con agua

* Comunicación preliminar.

* Jefe de Residentes de Obstetricia y Ginecología.

$* *$ Instructor del Departamento de Fisiología.

$* * *$ Profesor Agregado del Departamento de Fisiología. Universidad de Antioquia. Facultad de Medicina. 
y jabón. La manera de colocar los electrodos se indica en la figura No 1 . Se toman registros unipolares con referencia a tierra y bipolares entre pares de electrodos vecinos.

Es muy importante que la vejiga se encuentre vacía, para evitar interferencias. La paciente se coloca en decúbito dorsal, solicitándole la mayor relajación posible. En algunos casos especiales se obtienen mejores registros sentando la paciente.

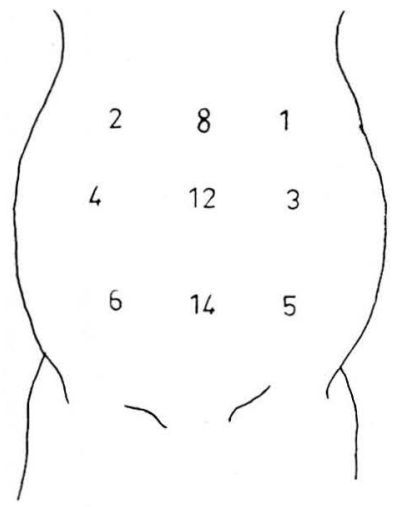

E.C.G.F; COLOCACION DE ELECTRODOS

figura 1

Los requisitos mínimos que tuvimos en cuenta para considerar positivo un electrocardiograma fetal fueron los siguientes:

1. Que la onda R del complejo fetal se observe durante todo el trazado.
2. Que dicha onda aparezca a intervalos de tiempo regulares.

Aun cuando ciertos autores han informado algunas anormalidades del corazón fetal, determinadas por este método, no hemos utilizado el electrocardiograma con este propósito, sino para saber si existe o no feto vivo.

\section{Resultados}

Se practicaron 26 E. C. G. F. en 25 pacientes. La edad estaba comprendida entre los 16 y los 39 años; cuatro eran primigestantes y las restantes multíparas. La distribución de la edad del embarazo se aprecia en el siguiente cuadro:

\begin{tabular}{cc}
\hline $\begin{array}{c}\text { Número } \\
\text { de pacientes }\end{array}$ & $\begin{array}{c}\text { Edad del } \\
\text { embarazo en meses }\end{array}$ \\
\hline 1 & 3 \\
1 & $31 / 2$ \\
4 & 4 \\
3 & 5 \\
1 & $51 / 2$ \\
2 & 6 \\
1 & $61 / 2$ \\
2 & 7 \\
1 & $71 / 2$ \\
4 & 8 \\
5 & 9 \\
\hline
\end{tabular}


El diagnóstico en doce pacientes fue embarazo normal; de éstas, siete con registro positivo, cinco tuvieron partos espontáneos con fetos vivos, las dos restantes, no han llegado a término. De las cinco pacientes con E. C. G. f. negativo, una tuvo un feto vivo, pero se encontró una alta resistencia eléctrica de la piel al practicar el registro. Las otras cuatro con trazados negativos están pendientes.

Una paciente tenía el diagnóstico de embarazo gemelar; en el registro sólo se encontró un foco que indicaba presentación cefálica; posteriormente nacieron gemelos en buenas condiciones.

Dos tenían la sospecha diagnóstica de aborto frustrado, en una el E. C. G. f. fue negativo, y la paciente expulsó posteriormente un feto macerado; en la otra el registro fue positivo, y se encuentra actualmente en su sexto mes de embarazo.

En cinco, se encontró el diagnóstico de feto muerto. De ellas, tres con E. C. G. f. negativo, expulsaron fetos macerados; una con registro positivo, se encuentra en el séptimo mes de embarazo; en la otra paciente el registro fue indeciso, y a los diez días expulsó un feto macerado en presentación podálica, con tres circulares del cordón.

Con el diagnóstico de mola hidatidiforme se encontraron tres pacientes; de éstas, dos presentaron registros positivos, y se encuentran en su cuarto y quinto mes de embarazo respectivamente. La otra, con trazado negativo, expulsó una mola.

Dos pacientes tenían el diagnóstico de amenaza de aborto; en una el E. C. G. f. fue positivo, y se encuentra en el quinto mes de embarazo. En la otra el primer registro fue positivo, pero posteriormente dejó de sentir movimientos fetales; se repitió el E. C. G. f., fue negativo; esta paciente expulsó luego un feto macerado.

En la última paciente con cinco $y$ medio meses de embarazo y sospecha de pielonefritis, el E. C. G. f. fue positivo. 
TABLA I

\begin{tabular}{|c|c|c|c|c|c|c|c|c|c|}
\hline $\begin{array}{l}\text { No de } \\
\text { Orden }\end{array}$ & Edad & Gravidez & $\begin{array}{c}\text { Mes de } \\
\text { embarazo }\end{array}$ & $\begin{array}{r}\text { Ruidos } \\
\text { fetales }\end{array}$ & Presentación & $\begin{array}{l}\text { Diagnóstico } \\
\text { clínico }\end{array}$ & $\begin{array}{l}\text { E. C. } \\
\text { Result. }\end{array}$ & $\begin{array}{l}\text { s. f. } \\
\text { Frec. }\end{array}$ & Observaciones \\
\hline 1 & 29 & 7 & 9 & + & Cefálica & $\begin{array}{l}\text { Embarazo } \\
\text { normal }\end{array}$ & $t-$ & 164 & Nació feto vivo \\
\hline 2 & 39 & 8 & 8 & $-i-$ & $\begin{array}{l}\text { Cefálica } \\
\text { podálica }\end{array}$ & $\begin{array}{l}\text { Doble foco. } \\
\text { Gemelar }\end{array}$ & \pm & 151 & $\begin{array}{l}\text { Nacen gemelos } \\
\text { vivos }\end{array}$ \\
\hline 3 & 37 & 11 & 8 & + & Cefálica & $\begin{array}{l}\text { Ruptura precoz } \\
\text { de membranas }\end{array}$ & - & & Nació feto vivo \\
\hline 4 & 30 & 8 & 6 & - & - & Feto muerto? & + & 150 & Pendiente \\
\hline 5 & 33 & 6 & $61 / 2$ & - & - & Feto muerto & - & - & Expulsó feto muerto \\
\hline 6 & 28 & 7 & 8 & + & Cefálica & $\begin{array}{l}\text { Extrasístoles } \\
\text { fetales? }\end{array}$ & + & 150 & Nació feto vivo \\
\hline 7 & 20 & 1 & 5 & - & - & Mola & + & 156 & Pendiente \\
\hline 8 & 21 & 1 & 9 & - & Cefálica & $\begin{array}{l}\text { Dejó de sentir } \\
\text { movimientos } \\
\text { fetales } 2 \\
\text { días antes }\end{array}$ & - & 一 & $\begin{array}{l}\text { Inducción. Nació } \\
\text { feto muerto. } \\
\text { Mongol }\end{array}$ \\
\hline
\end{tabular}


TABLA II

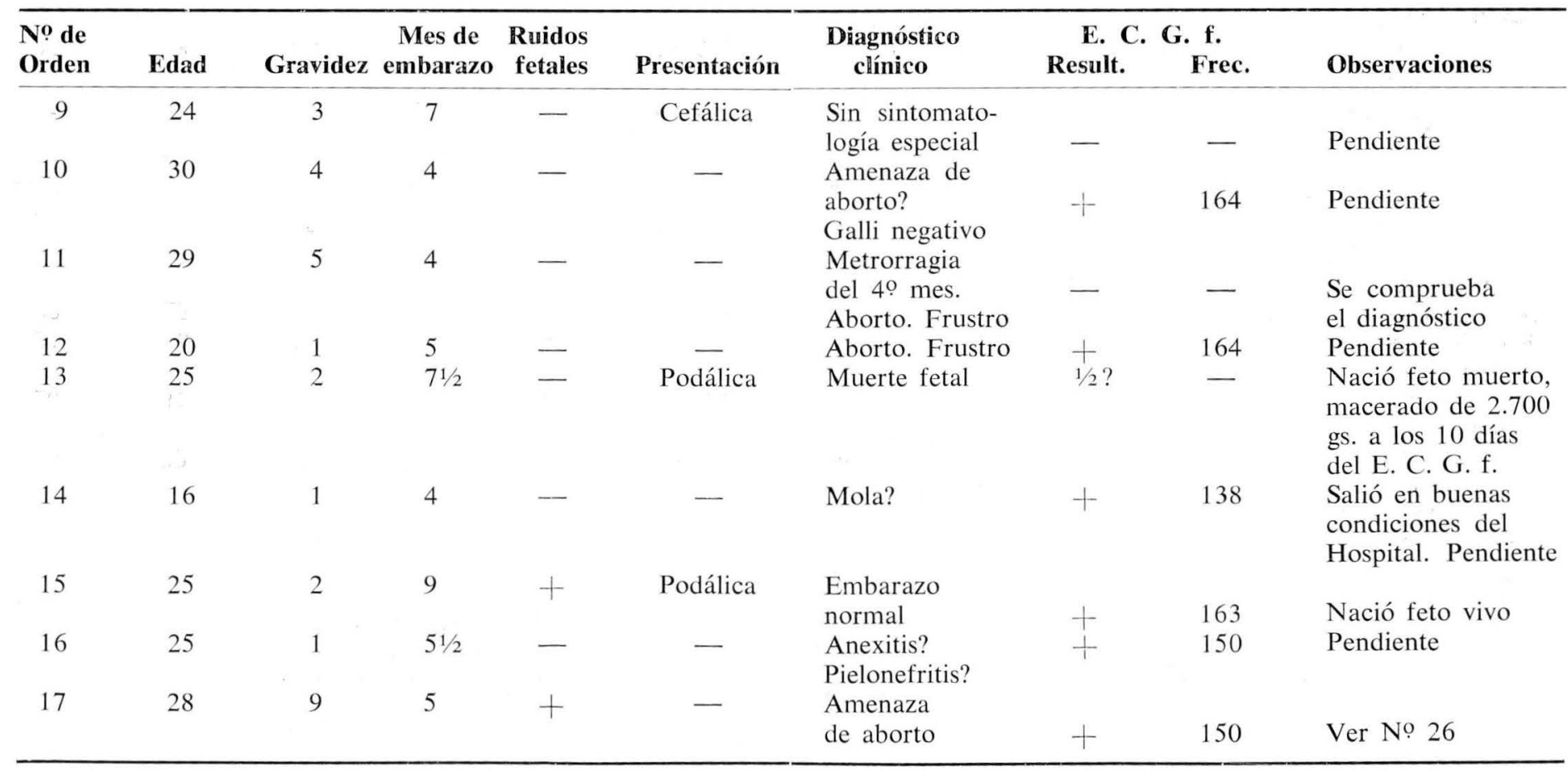




\section{TABLA III}

\begin{tabular}{|c|c|c|c|c|c|c|c|c|c|}
\hline $\begin{array}{l}\text { No de } \\
\text { Orden }\end{array}$ & Edad & Gravidez & $\begin{array}{l}\text { Mes de } \\
\text { embarazo }\end{array}$ & $\begin{array}{r}\text { Ruidos } \\
\text { fetales }\end{array}$ & Presentación & $\begin{array}{l}\text { Diagnóstico } \\
\text { clínico }\end{array}$ & $\begin{array}{l}\text { E. C. } \\
\text { Result. }\end{array}$ & $\begin{array}{l}\text { G. f. } \\
\text { Frec. }\end{array}$ & Observaciones \\
\hline 18 & 31 & 4 & 7 & + & Cefálica & $\begin{array}{l}\text { Embarazo } \\
\text { normal }\end{array}$ & - & - & $\begin{array}{l}\text { Altísima resistencia } \\
\text { eléctrica de la piel. } \\
\text { Sale sin tener } \\
\text { el parto }\end{array}$ \\
\hline 19 & 32 & 9 & 9 & + & Cefálica & $\begin{array}{l}\text { Embarazo } \\
\text { normal }\end{array}$ & + & 153 & Nació feto vivo \\
\hline 20 & 31 & 4 & 9 & + & Cefálica & $\begin{array}{l}\text { Embarazo } \\
\text { toxemia }\end{array}$ & + & 130 & Nació feto vivo \\
\hline 21 & 36 & 10 & 3 & - & - & Mola? & - & - & Expulsó una mola \\
\hline 22 & 25 & 3 & 6 & + & Cefálica & Embarazo & - & - & $\begin{array}{l}\text { Pendiente. Se repite } \\
\text { el electro, y es } \\
\text { negativo }\end{array}$ \\
\hline 23 & 29 & 5 & 8 & + & Cefálica & $\begin{array}{l}\text { Embarazo } \\
\text { normal }\end{array}$ & $-1-$ & 153 & Pendiente \\
\hline 24 & 29 & 4 & $31 / 2$ & + & & $\begin{array}{l}\text { Embarazo } \\
\text { normal }\end{array}$ & - & - & Pendiente \\
\hline 25 & 23 & 6 & 4 & + & Cefálica? & $\begin{array}{l}\text { Embarazo } \\
\text { normal }\end{array}$ & + & 150 & Pendiente \\
\hline 26 & 28 & 9 & 5 & - & Cefálica & $\begin{array}{l}\text { Dejó de sentir } \\
\text { movimientos } \\
\text { R. F. negativos }\end{array}$ & - & - & Nació feto macerado \\
\hline
\end{tabular}




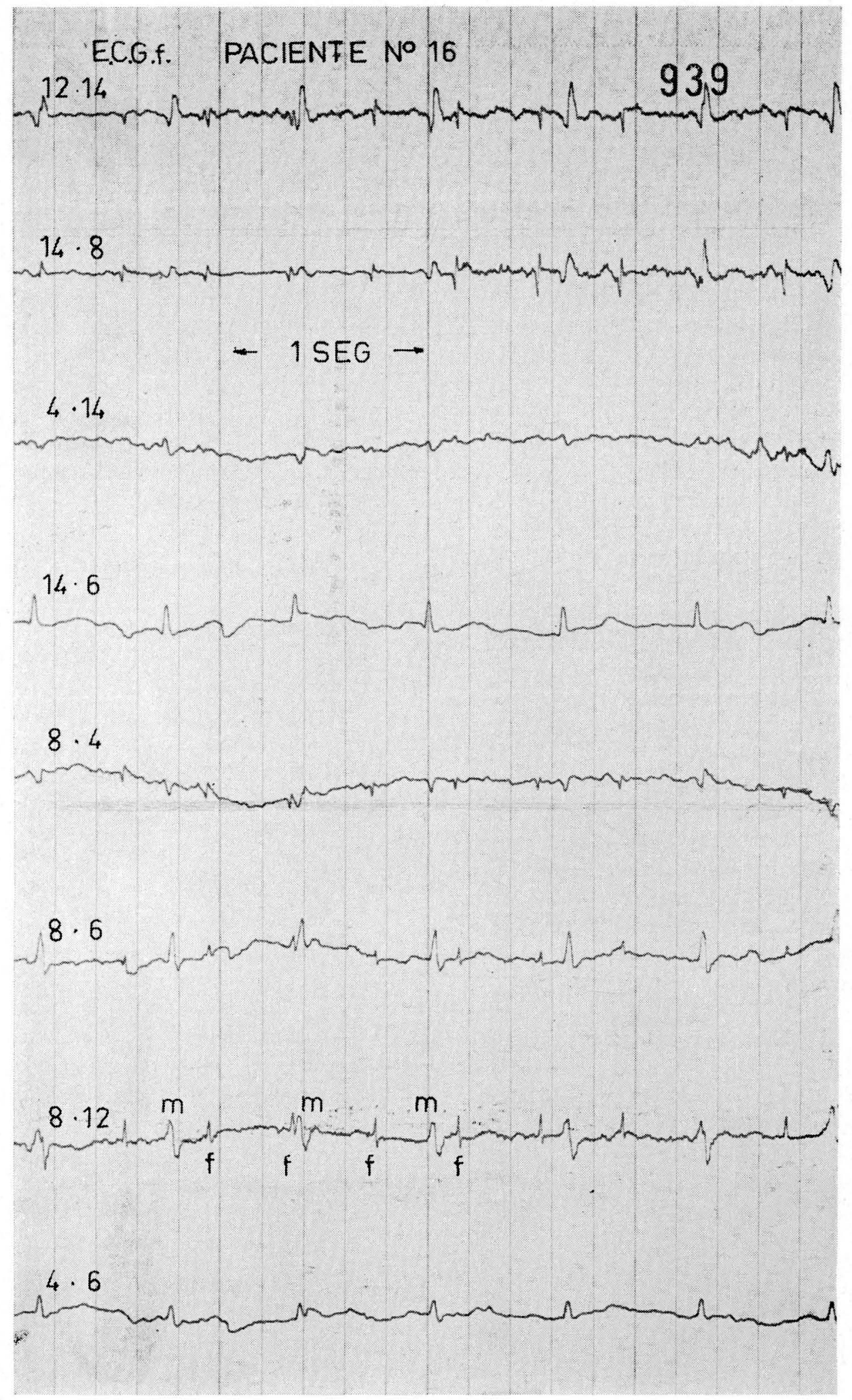

Figura 2 


\section{Comentarios y conclusiones}

En el presente estudio no intentamos investigar anomalías congénitas del corazón fetal ni anoxia in útero; consideramos el E. C. G. f. como un método sencillo y de gran valor en los casos en los cuales se sospecha muerte fetal.

¿Cuál es el valor práctico del electrocardiograma fetal y está justificada su utilización en todas las unidades obstétricas? El valor del E. C. G. f. está comprobado en la práctica obstétrica; cuando la viabilidad del feto está en duda, un trazado positivo es concluyente y definitivo. Es lógico pensar que el costo del equipo y el sueldo de un ingeniero eléctrico hacen difícil la práctica del método en hospitales pequeños.

El hallazgo de trazados negativos en algunos casos no indica muerte fetal, debido posiblemente a que el corazón fetal presenta una actividad eléctrica mucho menor, en relación con los distintos tipos de interferencias eléctricas encontradas al tomar el registro.

La diferencia entre mola hidatidiforme y embarazo normal puede ser determinada cuando un trazado electrocardiográfico es positivo. Aun cuando teóricamente el E. C. G. f. puede encontrarse hacia los 30 días de gestación, tiempo en el cual ocurre la fusión del tubo cardíaco en el embrión humano, todos los autores están de acuerdo que puede obtenerse un registro positivo a las doce semanas.

La presentación cefálica o podálica pueden ser diferenciadas fácilmente, relacionadas con la dirección del complejo materno. En el primer caso el eje eléctrico del corazón fetal apunta en dirección contraria al eje eléctrico de la madre; en el segundo caso ambos tienen la misma dirección.

\section{CONCLUSIONES}

1. Se estudiaron 25 pacientes, a las cuales se les practicó 26 E. C. G. f.

2. Se presenta una técnica sencilla y práctica, la cual es aplicable especialmente en el último trimestre del embarazo.

3. Los resultados se obtienen inmediatamente con un mínimo de interpretación.

4. Con el E. C. G. f. se puede hacer el diagnóstico de feto vivo, diferenciar una mola del embarazo normal y facilitar el reconocimiento de la presentación.

5. El E. C. G. f. puede aparecer a las doce semanas de gestción. 